\title{
Benign Peripheral Nerve Sheath Tumors Presenting as Small, Tender Scalp Masses
}

\author{
Corresponding author: Byung-chul Son \\ Department of Neurosurgery, Seoul St. \\ Mary's Hospital, College of Medicine, \\ The Catholic University of Korea, 222 \\ Banpo-daero, Seocho-gu, Seoul 06591, \\ Republic of Korea \\ Tel: +82-2-2258-6122 \\ Fax: $+82-2-594-4248$ \\ E-mail: sbc@catholic.ac.kr
}

Sol-hooy Oh${ }^{1}$, Hak-cheol Ko ${ }^{1}$, Jin-gyu Choi ${ }^{1}$, Byung-chul Son ${ }^{1,2}$

${ }^{1}$ Department of Neurosurgery, Seoul St. Mary's Hospital, College of Medicine, The Catholic University of Korea, Seoul, ${ }^{2}$ Catholic Neuroscience Institute, College of Medicine, The Catholic University of Korea, Seoul, Republic of Korea

Received: June 3, 2017

Revised: July 15, 2017

Accepted: July 21, 2017
Benign peripheral nerve sheath tumors (PNSTs) such as neurofibromas and schwannomas may present with extracranial head and neck mass. However, their presentation with simple scalp mass is rare. Here, we report two cases of PNSTs presented as small and tender scalp masses. One was a solitary neurofibroma while the other was schwannoma by pathological examination through excisional biopsy. Both occurred in women in their 50s without any evidence of neurofibromatosis. Although lesions initially presented as scalp mass are quite common entities with a wide variety of causes, such lesions with clinically significant pathologies are not negligible. They need long-term follow-up and further treatment. Therefore, small PNSTs should be included in the differential diagnosis of scalp mass that shows typical radiating paresthesia on palpation in the distribution of the occipital nerve.

Key Words: Neoplasms; Nerve sheath neoplasms; Neurofibroma; Scalp; Schwannoma

\section{INTRODUCTION}

Patients presenting with scalp mass are frequently encountered in daily practice ${ }^{4)}$. The spectrum of causes is comprehensive. They might be directly related to the scalp itself. They might be secondary stigmata of an underlying process in the skull. The overall incidence of specific diagnosis and rates of clinically significant lesions presenting as scalp masses have not been well-defined ${ }^{4)}$. According to a study to determine the incidence of scalp mass in adults ${ }^{4)}$, the five most common histological diagnosis are trichilemmal cyst, epidermal cyst, lipoma, nevus, and sebaceous cyst. The rate of "clinically significant" pathologies meaning malignancies or those that need follow-up is approximately $7.8 \%{ }^{4}$. Scalp masses originated from neural components included neurofibroma and Merkel cell carcinoma, although their incidence is quite low $(0.6$ and $0.3 \%$, respectively $)^{4)}$. Preoperative diagnosis of scalp mass is difficult ${ }^{4)}$.

Benign peripheral nerve sheath tumors (PNSTs) such as neurofibromas and schwannomas may present as extracranial head and neck mass ${ }^{3}$. However, their presentation as small and tender scalp mass is extremely rare. Here, we report two cases of PNSTs presented as small and tender scalp masses.

\section{CASE REPORTS}

\section{Case 1}

A 56-year-old female presented a small and palpable tenderness on her left temporal area. Until feeling electricity-like pain and tenderness in her left temporal area, she did not realize the presence of this lesion 2 months before presentation. There was no spontaneous pain or headache. A brief episode of electricity-like pain was elicited when touching the mass. It was movable, showing rubbery in consistency. Her medical and family history was unremarkable. Her neurologic examination was normal. There was no hypesthesia or paresthesia around the mass. No abnormal skin pigmentation or café au lait spot was observed. An excision biopsy was performed and a $3 \times 8 \mathrm{~mm}$-sized whitish mass was excised. Histologic examination revealed a neurofibroma (Fig. 1). No recurrence was observed with 12 months of follow-up.

\section{Case 2}

A 56-year-old female presented a small tenderness in her right retroauricular occipital area for three months. She realized the presence of a small palpable mass in her occipital scalp 
one year prior to presentation. It showed gradual enlargement. There was no pain or tenderness at the beginning. However, aching pain and tenderness gradually developed. At one and a half years prior to presentation, she underwent an excisional biopsy for a small tender scalp mass adjacent to the location of the present one. At that time, no histologic examination was performed. Her postoperative course was uneventful. Due to increasing pain intensity and progressive enlargement of her occipital mass, she was referred for histologic diagnosis.

Her neurologic examination was normal. Her family and medical history were unremarkable. Manipulation of the mass elicited an incident and sharp pain over the mass with radiating paresthesia over the parieto-occital scalp. There was no café au lait spot or other skin stigmata of neurofibromatosis. An excisional biopsy revealed a $4 \times 10 \mathrm{~mm}$-sized, fusiform swelling of a small branch of the lesser occipital nerve (Fig. 2A). The mass was excised en-bloc. Histologic examination revealed a schwannoma. Immunostaining for S-100 was positive. Neuro-

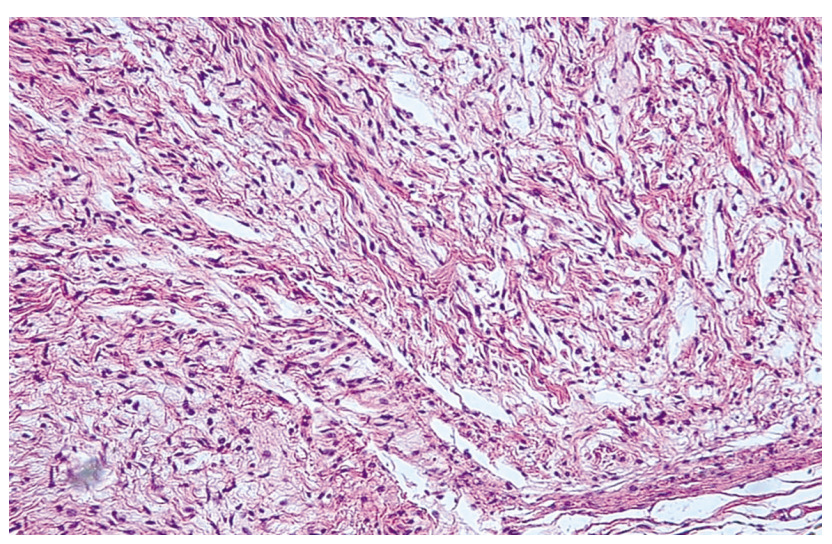

Fig. 1. Histologic finding of peripheral nerve sheath tumor presenting with small tender scalp mass (case 1). Histological examination showed a loose pattern of spindle-shaped cells with elongated wavy nuclei set in myxoid stroma with wire-like collagen fiber network indicating neurofibroma (hematoxylin and eosin stain [H \& E], ×100 magnification). filament was negative with a localized focus of increased Ki-67 (up to 15\% was noted within the mass). Mild paresthesia was observed in her parieto-occipital scalp at 3 months postoperatively. There was no recurrence or new occurrence of scalp mass at the last follow-up at postoperative 12-month.

\section{DISCUSSION}

Differential diagnosis and management strategies for scalp masses have not been described in details yet. Their clinical significance also remains unclear. It is difficult to refer to the literature regarding the overall incidence of particular diagnosis and rates of clinically significant lesions presenting as scalp masses $^{4}$. Scalp masses have been underestimated. Interdisciplinary consultations have not been performed for scalp masses comprehensively ${ }^{4}$. According to a recent study in a single center $^{4}$, the overall incidence of clinically significant pathologies of scalp mass is around $7.8 \%$. The incidence of correct preoperative diagnosis for scalp mass is only 13 to $27 \%{ }^{4}$ ). Preoperative diagnosis of scalp mass is difficult ${ }^{4)}$. It is not specified by the surgeon in $53 \%$ of patients. Even if it is specified preoperatively, preoperative diagnosis is correct in just 13 to $27 \%$ of these cases with respect to surgical specialties ${ }^{4}$.

In the report of Türk et al. ${ }^{4)}$, for 345 patients with scalp masses, the most common pathology is found to be trichilemmal cyst, followed by epidermal cyst, lipoma, nevus, and sebaceous cyst. There five diagnoses account for $77.1 \%$ of these cases ${ }^{4)}$. Tumors originating from cutaneous appendages (trichilemmal cyst, pilomatrixoma, sebaceous cyst, sebaceous carcinoma, apocrine mixed tumor, syringocystadenoma, hidradenoma, eccrine cell carcinoma, and nevus sebaceous) have constituted almost half of these cases $(49 \%)^{4)}$. A total of $82.3 \%$ of these lesions have originated from skin components, the epidermis, or cutaneous appendages ${ }^{4}$. The rest lesions are tumors of fat tissue, muscles, cartilage, bones, neural and neuroendocrine components, vascular tissue, proliferation of fibrous or related tissues,
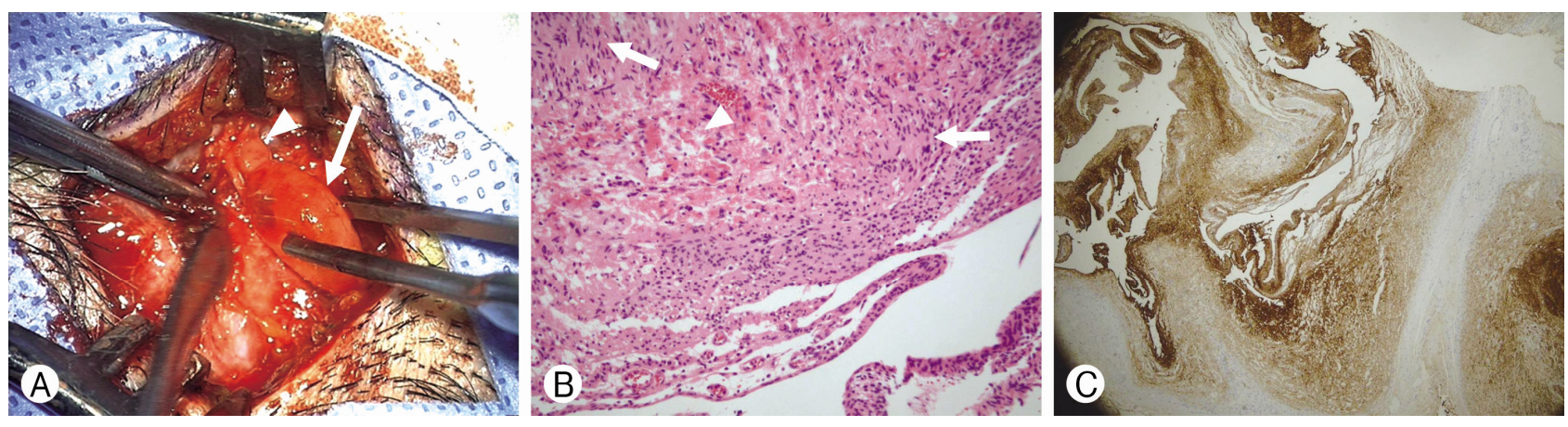

Fig. 2. Intraoperative finding and histologic specimen of schwannoma presenting as small scalp mass (case 2). (A) An intraoperative photograph showing schwannoma (arrow) originated from a distal branch of the occipital nerve (arrowhead). (B) Histologic analysis showing a characteristic fin- ding of cellular and spindled Antoni A areas (arrows) with distinctive nuclear palisading (Verocay bodies) and looser, myxoid Antoni B areas (arrowhead, hematoxylin and eosin stain [H \& E], $\times 40$ magnification). (C) Positive stain with S-100 protein ( $\times 40$ magnification) indicating schwannoma. 
and cutaneous metastases or cutaneous lymphoid infiltrations. In their series, there were $2(0.6 \%)$ cases of neurofibroma without any case of schwannoma. Although huge scalp masses due to PNSTs have been sporadically reported, their presentation as small and tender scalp mass is rare.

Among benign tumors of peripheral nerves, one specific group originated from Schwann cells is currently divided into two subtypes: neurofibromas and schwannomas ${ }^{3)}$. Schwannomas also arise from encapsulated masses on the periphery of nerves. They contain characteristic cellular and spindled Antoni A areas with distinctive nuclear palisading(Verocay bodies) and looser Antoni B areas ${ }^{2)}$. Entrapped axons are not their common features. In contrast, neurofibromas will diffusely expand the involved nerve and encompass entrapped axons. Schwannomas commonly arise from intracranial nerves while neurofibromas rarely affect cranial nerves. Neurofibromas may undergo malignant degeneration while schwannomas very rarely do $\mathrm{so}^{5)}$.

Schwannomas occurring in the head and neck region account for approximately $25 \%$ of these cases. They are sometimes associated with von Recklinghausen's disease in 8 to $18 \%$ of cases. When cases are not associated with neurofibromatosis as depicted in the current report, schwannomas are clinically seen as solitary slow-developing lesions with symptoms only when large areas have been affected $^{1)}$. Presentation might be pain along the distribution of nerve, hypesthesia and tenderness. Extradural schwannomas are most commonly found in association with large nerve trunks where motor and sensory modalities are intermixed ${ }^{2)}$.

\section{CONCLUSION}

PNST such as neurofibroma and schwannoma may present as small tender scalp mass. They should be included in differential diagnosis of scalp mass.

\section{CONFLICTS OF INTEREST}

No potential conflict of interest relevant to this article was reported.

\section{REFERENCES}

1. Asuquo ME, Nwagbara VI, Akpan SO, Otobo FO, Umeh J, Ebughe $G$, et al.: Recurrent benign schwannoma of the scalp: Case report. Int J Surg Case Rep 4:65-67, 2013

2. Feany MB, Anthony DC, Fletcher CD: Nerve sheath tumours with hybrid features of neurofibroma and schwannoma: a conceptual challenge. Histopathology 32:405-410, 1998

3. Langner E, Del Negro A, Akashi HK, Araújo PP, Tincani AJ, Martins AS: Schwannomas in the head and neck: retrospective analysis of 21 patients and review of the literature. Sao Paulo Med J 125:220-222, 2007

4. Türk CÇ, Bacanlı A, Kara NN: Incidence and clinical significance of lesions presenting as a scalp mass in adult patients. Acta Neurochir (Wien) 157:217-223, 2015

5. Woodruff JM, Selig AM, Crowley K, Allen PW: Schwannoma (neurilemoma) with malignant transformation. A rare, distinctive peripheral nerve tumor. Am J Surg Pathol 18:882-895, 1994 